\title{
Theoretical Analyses and Experimental Evaluation of a Small-Displacement Sensor Based on Surface Plasmon Resonance Technology
}

\author{
Shinn-Fwu Wang*, Chi-Tun Chen, Fu-Hsi Kao, Yi Chu, \\ Yu-Pin Liao, Shyh-Rong Lay and An-Li Liu \\ Department of Electronic Engineering, Chien Hsin University of Science and Technology, \\ No. 229, Jianxing Rd., Zhongli City, Taoyuan County 32097, Taiwan
}

(Received December 24, 2013; accepted March 6, 2014)

Key words: small-displacement measurement, surface plasmon resonance (SPR), heterodyne interferometry (HI), Kretchmann's configuration

In this paper, a small-displacement sensor based on the surface plasmon resonance technology and heterodyne interferometry is proposed. The basic sensing unit is composed of a prism assembly and a displacement probe. The prism assembly consists of a halfwave $(\lambda / 2)$ plate, two right-angle prisms with two metal films, and two rotation stages. The small-displacement sensor has high sensitivity and resolution owing to the attenuated total reflection effect in heterodyne interferometry. Additionally, we can obtain the results of the experiment in a distant place by using of a ZigBee module and a USB data acquisition card (DAQ card). It can be seen that the displacement resolution of the small-displacement sensor can reach $0.155 \mathrm{~nm}$. The small-displacement sensor has some merits, such as easy operation, high measurement accuracy, high resolution, and rapid measurement, and its feasibility has been demonstrated.

\section{Introduction}

Owing to the importance of small-displacement measurement in the fields of high precision industries, there have been many articles published in recent years. ${ }^{(1-5)}$ It is well known that the phenomenon of surface plasmon resonance (SPR) in thin metal films is highly sensitive to the optical and structural properties of the metal interface. ${ }^{(6)}$ Among the studies, ${ }^{(1-5)}$ researchers focused on biochemical and biological sensors, ${ }^{(7)}$ and very few attempts have been made at other applications, such as small-angle measurement and small-displacement measurement. At this moment, a special application of the SPR technology is proposed. On the basis of the issue of small-displacement measurement, a prism assembly ${ }^{(8)}$ consisting of a halfwave $(\lambda / 2)$ plate, two right-angle prisms with

"Corresponding author: e-mail: sfwangking@gmail.com, sfwang@uch.edu.tw 
two metal films, and two rotation stages is used as the basic sensing unit in the smalldisplacement sensing system.

It is worth mentioning that the hypotenuses of two right-angle prisms have been coated with a $2 \mathrm{~nm}$ Ti film and a $45.5 \mathrm{~nm}$ Au film. However, the small-displacement sensor has some merits, such as easy operation, high measurement accuracy, high resolution, and rapid measurement. Moreover, the experimental results and theoretical curve show good correspondence.

\section{Principles}

A four-layer device [prism-titanium (Ti)-gold ( $\mathrm{Au}$ )-air] of Kretchmann's configuration ${ }^{(9)}$ is shown in Fig. 1. In fact, the surface plasmons are excited when the wave vector of incident light, $k_{\mathrm{x}}$, and the real part of the surface plasmon wave vector, $k_{\mathrm{sp}}$, satisfy the matching condition given by

$$
k_{\mathrm{x}}=k_{0} n_{1} \sin \alpha=\frac{2 \pi}{\lambda_{0}} n_{1} \sin \alpha=\operatorname{Re}\left\{k_{\mathrm{sp}}\right\}
$$

where $k_{0}$ is the free-space wave number, $n_{1}$ the refractive index of the prism, $\alpha$ the angle of incidence, and $\lambda_{0}$ the wavelength of light in free space. At this moment, $\alpha=\alpha_{\mathrm{sp}}$ is called the resonance angle. If the amplitude reflection coefficients $r_{1234}^{\mathrm{p}}$ and $r_{1234}^{\mathrm{s}}$ are written as

$$
r_{1234}^{\mathrm{p}}=\left|r_{1234}^{\mathrm{p}}\right| e^{i \varphi_{\mathrm{p}}}, r_{1234}^{\mathrm{s}}=\left|r_{1234}^{\mathrm{s}}\right| e^{i \varphi_{\mathrm{s}}},
$$

then the phase difference variation $\delta$ between $\mathrm{p}$ - and s-polarization components is

$$
\delta=\varphi_{\mathrm{p}}-\varphi_{\mathrm{s}}
$$

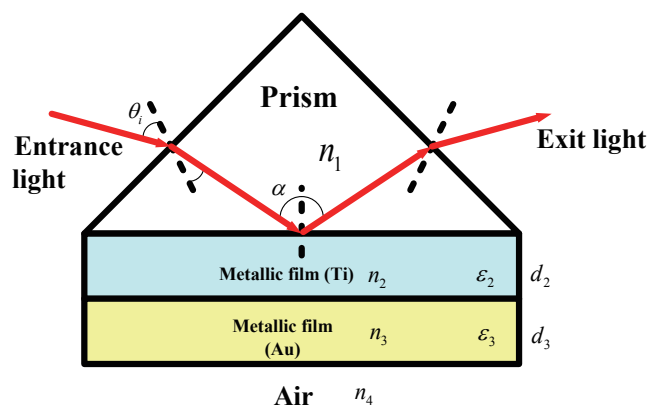

Fig. 1. (Color online) Four-layer Kretchmann's configuration $\left(n_{1}, n_{2}, n_{3}\right.$, and $n_{4}$ are the refractive indices of glass, Ti, $\mathrm{Au}$, and air, respectively. $d_{2}$ and $d_{3}$ are the thicknesses of Ti and Au, respectively). 
As shown in Fig. 2, a displacement probe (DP) is used in the system. The displacement probe is composed of an objective lens with a focal length $f$ and a mirror located near the focal plane of an objective lens. As a light ray is incident on the objective lens, it is reflected by the mirror later and then the reflected light passes through the objective lens again. If the mirror is located at the focal plane, it will be in the antiparallel direction with the incident beam. Otherwise, it will have a deviation angle $\Delta \theta$ with the antidirection of the incident beam. By using the refraction ray-tracing method, we can have

$$
\Delta z \simeq-\frac{f^{2}}{D} \Delta \theta
$$

From eq. (4), it is clear that the displacement $\Delta z$ is almost proportional to $\Delta \theta$. In this study, a prism assembly consisting of a halfwave $(\lambda / 2)$ plate, two right-angle prisms, and two rotation stages is used as the basic sensing unit in the small-displacement sensing system, as shown in Fig. 3. If a p-polarization light enters the prism assembly, we can obtain the electric field of the exit light from the prism assembly by using the Jones matrix according to

$$
\begin{aligned}
\boldsymbol{E}_{\text {out }}=\boldsymbol{R}(-\theta) \cdot \boldsymbol{A} \cdot \boldsymbol{R}(\theta) \cdot \boldsymbol{E}_{\text {in }} & =\left[\begin{array}{cc}
\cos \theta & -\sin \theta \\
\sin \theta & \cos \theta
\end{array}\right]\left[\begin{array}{cc}
1 & 0 \\
0 & -1
\end{array}\right]\left[\begin{array}{cc}
\cos \theta & \sin \theta \\
-\sin \theta & \cos \theta
\end{array}\right] \cdot \boldsymbol{E}_{\text {in }} \\
& =\left[\begin{array}{cc}
\cos 2 \theta & \sin 2 \theta \\
\sin 2 \theta & -\cos 2 \theta
\end{array}\right]\left[\begin{array}{l}
1 \\
0
\end{array}\right]=\left[\begin{array}{c}
\cos 2 \theta \\
\sin 2 \theta
\end{array}\right],
\end{aligned}
$$

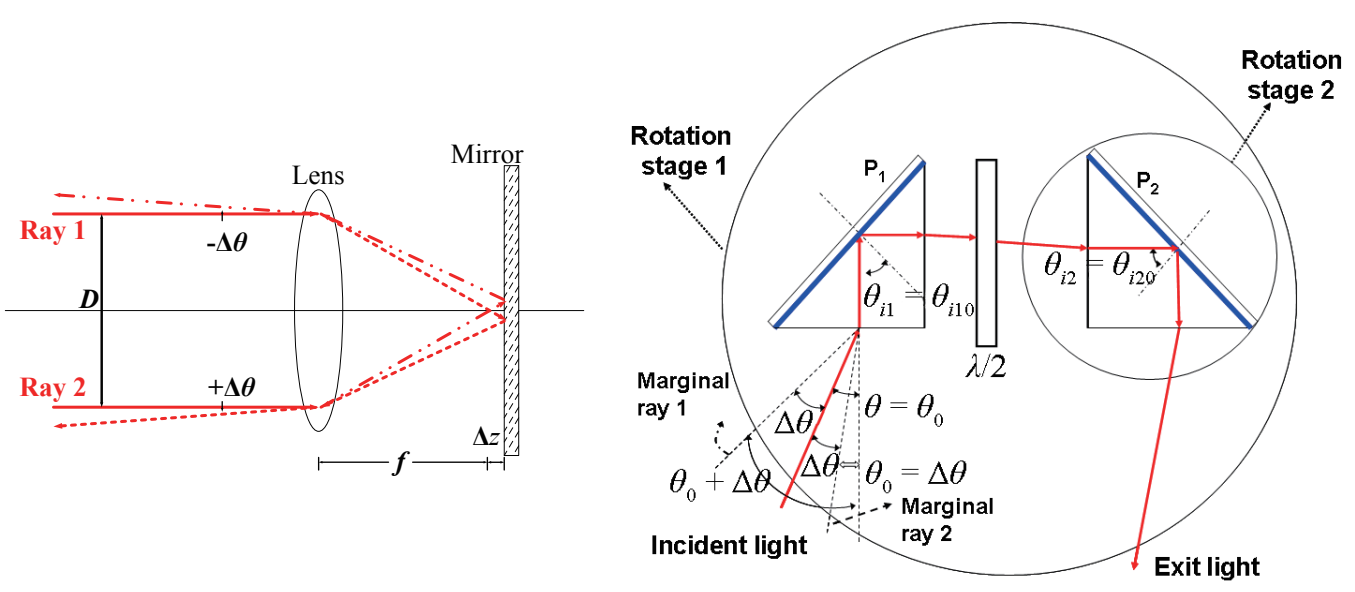

Fig. 2 (left). (Color online) Displacement probe.

Fig. 3 (right). (Color online) Prism assembly (basic sensing unit). 
where $\boldsymbol{A}$ is the Jones matrix of the halfwave $(\lambda / 2)$ plate, $\boldsymbol{E}_{\text {in }}=\left[\begin{array}{l}1 \\ 0\end{array}\right]$ is the Jones vector of the p-polarization light, and $\theta$ is the inclination angle of the fast axis of the halfwave $(\lambda / 2)$ plate with respect to the $x$-axis. As $\theta$ is equal to $45^{\circ}$, the exit light from the prism assembly is given by

$$
\boldsymbol{E}_{\text {out }}=\left[\begin{array}{c}
\cos 2 \theta \\
\sin 2 \theta
\end{array}\right]=\left[\begin{array}{c}
\cos 90^{\circ} \\
\sin 90^{\circ}
\end{array}\right]=\left[\begin{array}{l}
0 \\
1
\end{array}\right]
$$

It is clear that a p-polarization light is converted to an s-polarization light and vice versa. Suppose that the defocusing amount $\Delta z$ exists in Fig. 2, the reflected light coming from the DP is convergent or divergent. If the reflected light is divergent, the incident angle on the hypotenuse of the right-angle prism is not unique. In Fig. 3, the incident angles of the two marginal rays $(1 \& 2)$ that lie on the plane perpendicular to one side surface of the right-angle prism $\mathrm{P} 1$ are $\theta_{1}=\theta_{0}+\Delta \theta$ and $\theta_{2}=\theta_{0}-\Delta \theta$, respectively, where $\theta_{0}$ is the initial incident angle as $\Delta z=0$. It must be noted that the initial incident angle $\theta_{0}$ is chosen when the incident angle $\alpha$ is equal to the resonant angle $\alpha_{\mathrm{sp}}$, i.e., $\theta_{0}=\sin ^{-1}\left[n_{1} \sin \left(\alpha_{\mathrm{sp}}-45^{\circ}\right)\right]$, where $\alpha_{\mathrm{sp}}$ is almost equal to $43.813^{\circ}$ and $n_{1}$ is the refractive index of $\mathrm{P} 1$. If $\Delta z$ is equal to 0 , the two incident angles of the two prisms P1 and P2 must be adjusted to be equal, i.e., $\theta_{i 1}=\theta_{i 2}$. As $\Delta z \neq 0$, marginal ray 1 reflected from prism $\mathrm{P} 1$ will induce the phase difference variation $\Delta \phi_{1}$ between p- and s-polarization components and is given by $\left(\phi_{0}+\Delta \phi_{1}\right)-\phi_{0}=\Delta \phi_{1}$ (see Fig. 4). In the same way, the p- and s-polarization components reflected from P2 will induce the phase difference $\Delta \phi_{2}$ between the original p- and s-polarization components and is given by $\left(\phi_{0}-\Delta \phi_{2}\right)-\phi_{0}=-\Delta \phi_{2}$ (see Fig. 4). Because the p- and s-polarization components exchange, we have the phase difference $\Delta \phi=\Delta \phi_{1}+\Delta \phi_{2}$ as marginal ray 1 passes through the prism assembly. Similarly, marginal ray 2 will induce the phase difference $-\Delta \phi=-\left(\Delta \phi_{1}+\Delta \phi_{2}\right)$. Thus, the total phase difference variation $\Delta \phi_{\mathrm{t}}$ of the two marginal rays can be obtained and is given by $\Delta \phi_{\mathrm{t}}=2 \Delta \phi=2\left(\Delta \phi_{1}+\Delta \phi_{2}\right)$.

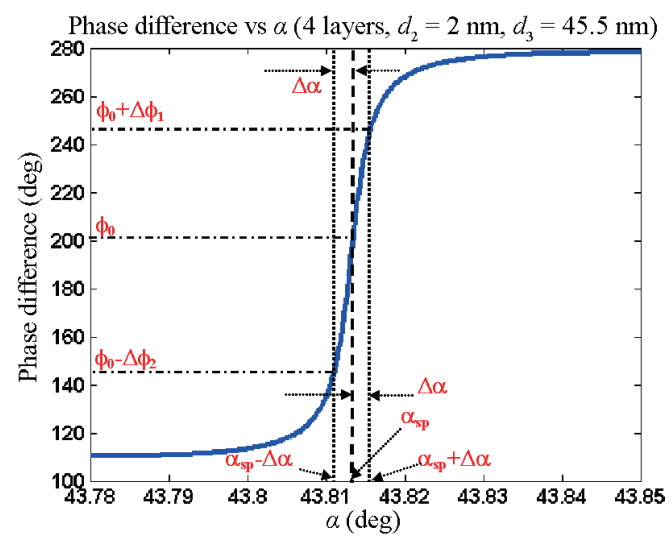

Fig. 4. (Color online) Phase difference vs incident angle. 


\section{Experiment and Results}

The experimental setup of the system is shown in Fig. 5. In addition, a heterodyne light source with $2 \mathrm{kHz}$ beat frequency is composed of a polarizer (PL), an electrooptical modulator (EOM), an electrooptical modulator driver, a function generator, and a laser diode of $635 \mathrm{~nm}$ wavelength as shown in Fig. 6. The light is incident on a beamsplitter $\mathrm{BS}$ and is divided into the reflected and transmitted lights. The transmitted light enters into the DP and is reflected back from the mirror. Afterwards, the light is reflected back

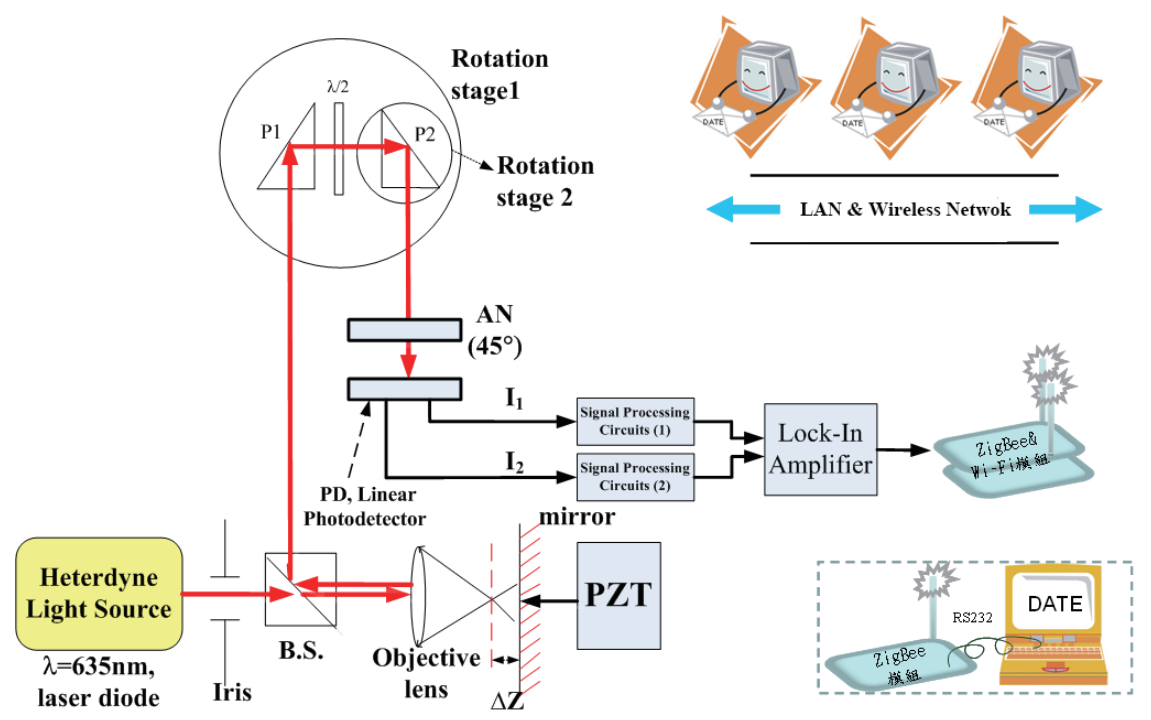

Fig. 5. (Color online) Experimental setup (B.S., beam splitter; PZT, piezoelectric; AN, analyzer).

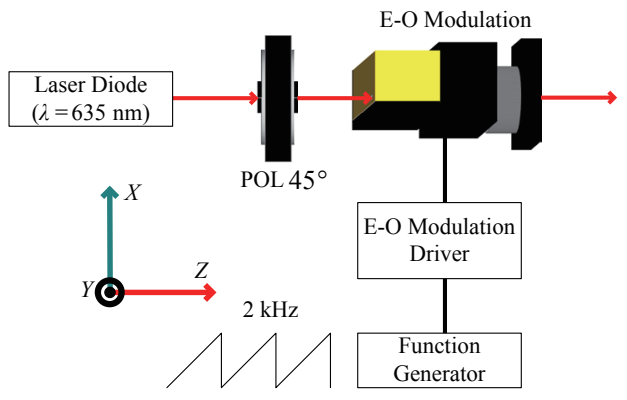

Fig. 6. (Color online) Optical structure of a heterodyne light source. 
to BS and enters the small-displacement sensor. Then, the other light passes through the prism assembly and an analyzer AN. Afterwards, it is detected by a linear photodetector. These two signals of two marginal rays detected by the linear photodetector are processed by signal processing circuits 1 and 2 . Finally, the two signals are sent to a lock-in amplifier (LIA) (SR830; manufactured by Stanford Research Systems, Sunnyvale, Calif.) with the resolution $\Delta \Phi$ of $0.01^{\circ}$. Thus, we can obtain the total phase difference variation $\Delta \phi_{\mathrm{t}}$ due to the ATR effect. After some numerical computations by a computer, the small displacement $\Delta z$ can be measured. Figure 7 shows variations in phase difference with the small displacement $\Delta z$. It is clear that the phase difference variation increases regularly as $\Delta z$ increases in the range of $-30 \mathrm{~nm} \leq \Delta z \leq 30 \mathrm{~nm}$.

\section{Discussion}

At this moment, let us discuss the sensitivity $S, S=\partial\left(\Delta \phi_{t}\right) / \partial(\Delta z)$, of the system. Figure 8 shows sensitivity as a function of small displacement $\Delta z$. It is evident that the sensitivity can reach $2.7 \% \mathrm{~nm}$. Additionally, let us discuss the resolution of the system: the resolution $R$ of the system is defined as

$$
R=\frac{\Delta \Phi}{S}
$$

where $\Delta \Phi$ is the resolution of the lock-in amplifier, $\Delta \Phi=0.01^{\circ}$. From Fig. 9 , it can be seen that the best resolution will reach $0.155 \mathrm{~nm}$ at least over the range of $-500 \mathrm{~nm} \leq \Delta z \leq 500 \mathrm{~nm}$. It is obvious that the sensitivity and resolution of the system are better than in the previous method, ${ }^{(10)}$ as shown in the theoretical and experimental results.

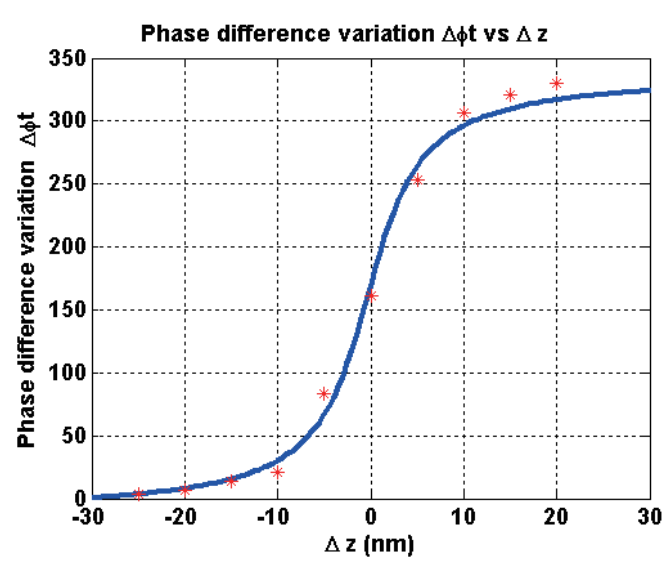

Fig. 7. (Color online) Experimental and simulation results. 

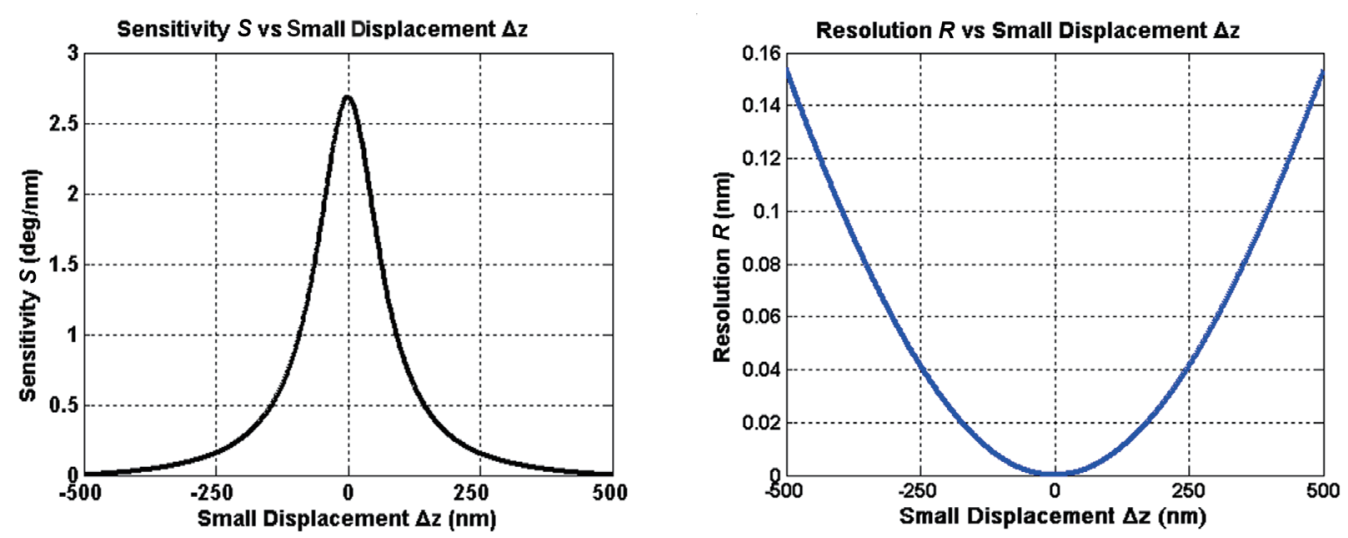

Fig. 8 (left). Sensitivity $S$ vs $\Delta z$.

Fig. 9 (right). (Color online) Resolution $R$ vs $\Delta z$.

\section{Conclusions}

In this paper, a small-displacement sensing system based on the surface plasmon resonance technology in heterodyne interferometry is proposed and evaluated. In fact, we also can obtain the results of the experiment in a distant place by using of a USB data acquisition card (DAQ card) and a ZigBee module, as shown in the experimental setup (see Fig. 5). Moreover, the sensitivity and resolution of the system can reach $2.7^{\circ} / \mathrm{nm}$ and 0.155 $\mathrm{nm}$, respectively. The small-displacement sensing system has some merits, such as easy operation, high measurement accuracy, high resolution, and rapid measurement, and its feasibility has been demonstrated.

\section{Acknowledgements}

This research was partially supported by the National Science Council of Taiwan through Grant Nos. NSC 100-2632-E-231-001-MY3 and NSC 99-2221-E-231-016.

\section{References}

1 A. L. Chaudhari and A. D. Shaligram: Sens. Actuators, A 100 (2002) 160.

2 H. Meng, W. Shen, G. Zhang, X. Wu, W. Wang, C. Tan and X. Huang: Sens. Actuators, B 160 (2011) 720.

3 J. Y. Lee, H. Y. Chen, C. C. Hsu and C. C. Wu: Sens. Actuators, A 137 (2007) 185.

4 K. H. Chen, H. S. Chiu, J. H. Chen and Y. C. Chen: Measurement 45 (2012) 1510.

5 K. Muthukkumaran and M.Gokul Krishnan: Int. J. Eng. Tech. Innovation 2 (2012) 31.

6 F. Meriaudeau, A. Wig, A. Passian, T. Downey, M. Buncick and T. L. Ferrell: Sens. Actuators, B 69 (2000) 51 .

7 R. Slavík, J. Homola and J. Čtyroký: Sens. Actuators, B 54 (1999) 74.

8 S. F. Wang, F. H. Kao, A. L. Liu, J. S. Chiu and W. Lai: IEEE I²MTC Proceedings (2011) 942.

9 E. Kretshmann: Z. Phys. 241 (1971) 313.

10 S. F. Wang: Sensors-Basel 9 (2009) 2498. 